九州大学学術情報リポジトリ

Kyushu University Institutional Repository

\title{
TERMINATING MARKOV RENEWAL PROCESSES IN VIEW OF LIFETIME MODEL OF RETREADED TIRES FITTED TO TRUCKS
}

Jiang, Yuanying

College of science, Guilin University of Technology

Endow, Yasushi

Department of Industrial Engineering, Chuo University

https://doi.org/10.5109/1434309

出版情報: Bulletin of informatics and cybernetics. 43，pp.23-39，2011-12. 統計科学研究会 バージョン：

権利関係 : 
TERMINATING MARKOV RENEWAL PROCESSES IN VIEW OF LIFETIME MODEL OF RETREADED TIRES FITTED TO TRUCKS

by

Yuanying JIANG and Yasushi Endow

Reprinted from the Bulletin of Informatics and Cybernetics

Research Association of Statistical Sciences, Vol.43

FUKUOKA, JAPAN

2011 


\title{
TERMINATING MARKOV RENEWAL PROCESSES IN VIEW OF LIFETIME MODEL OF RETREADED TIRES FITTED TO TRUCKS
}

\author{
By \\ Yuanying JIANG* and Yasushi ENDow ${ }^{\dagger}$
}

\begin{abstract}
The aim of this paper is to introduce a new type of terminating Markov renewal process $D(\alpha, \beta, k)$ in view of application to replacement problems. The dynamical sojourn probability and the renewal function of each Markov state, the time $\tau$ till termination, the distribution and moments of $\tau$ are calculated for this type terminating process in order to apply it to a lifetime model of replacement parts, such as retreadable tires fitted to trucks.
\end{abstract}

Key Words and Phrases: terminating Markov renewal process, sojourn time, terminating time, retread tires, replacement parts.

\section{Introduction}

Usually, a renewal problem is stated as follows: A given system starts with a new part or component, which fails at random time. A new part immediately enters the system to replace the first one and fails at another random time. There is another immediate replacement by a new part fitted in the system, still of the same type, and so on. But because most of artificial systems have their own lifetime, so Endow ( 2008) set up a terminal renewal model in which the process stops when the system comes to an end. This model was applied to predict numbers of replaced tires needed yearly in the Japanese motorcars market. In the terminal renewal process, the part replaced is assumed to be of the same grade or brand. However, there exist many systems in which parts are not necessarily replaced by the parts of the same grade or brand with which they were originally equipped. Endow and Tanimoto (2010) put forward a theory of Markov renewal process with termination, and applied it to a demand prediction model of motorcars tires in the market.

In the previous works mentioned above, the parts replaced are assumed to be of new ones, but trucking fleets and owners of commercial vehicles use both new and retread tires on their vehicles. So we introduce a new $D(\alpha, \beta, k)$-terminating Markov renewal process which models a lifetime of a truck tire stochastically. This kind of termination occurs when the same state is visited two or perhaps more times. An example of such

\footnotetext{
* College of science, Guilin University of Technology, Guilin 541004, P. R. China. e-mail: jyy@glite.edu.cn Yuanying Jiang was supported by the National Natural Science Foundation of China (11061012) and the Guangxi China Science Foundation (0991081, 2010GXNSFA013120).

$\dagger$ Department of Industrial Engineering, Chuo University 1-13-27 Kasuga Tokyo 112-8551 Japan. email: endow@indsys.chuo-u.ac.jp Yasushi Endow was supported by the Grants from Chuo University and Bridgestone Corporation.
} 
a process would be a machine or a piece of equipment in which various parts may fail at random times. The machine or equipment is retreaded or repaired but is discarded (the process ends) when the retread or repaired occurs, for example, a second time (or $K$ times). Many examples exist of this common type of termination including the replacement of truck's tires and the replacement of cutting tools fitted to machine tools. Of interest in this processes are the sojourn probability, renewal function of each state, and the time $\tau$ until termination, the distribution and moments of $\tau$, which are obtained for the $D(\alpha, \beta, k)$-terminating Markov renewal processes.

This paper is organized as follows. In Section 2, semi-Markov processes and Markov renewal processes are briefly reviewed for completeness of discussion. The renewal functions and the dynamical sojourn probability for each state are also given in the explicit forms. In Section 3, we introduce a terminating MRP of finite transition type, which stops after a prefix number of transitions, and apply it to lifetime model for retread tires fitted to trucks. In Section 4, a new type of MRP processes $D(\alpha, \beta, k)$ are introduced to apply a replacement problem, which is different from the previous one in stopping rules (see Endow and Tanimoto (2010)). The distribution and moments of $\tau$ are obtained for the $D(\alpha, \beta, k)$-terminating MRP's, explicitly. In Section 5 some concluding remarks are stated in practical view points.

\section{Markov Renewal Processes}

For the sake of completeness of discussion, let us briefly review theory of Markov renewal processes and state some results which will be of use later in the following section.

\subsection{Markov renewal processes}

Let $(Z, X):=\left\{\left(Z_{n}, X_{n}\right)\right\}_{n \geq 0}$ be a pair of stochastic processes defined on a complete probability space $(\Omega, \mathcal{F}, \mathcal{P})$ in which $Z$ is a Markov chain with a state space $I$ and a transition rule $(\mathbf{p}, P)$, as follows. The state space is $I=\{1,2, \cdots, m\}$, the initial probability vector $\mathbf{p}=\left(p_{1}, \cdots, p_{m}\right)$ with

$$
p_{i}:=\mathbf{P}\left\{Z_{0}=i\right\} \geq 0, i \in I ; \quad \sum_{i=1}^{m} p_{i}=1,
$$

and the sequence of transition probability matrices $P=\left\{P^{(n)}\right\}_{n \geq 1}$ with $P^{(n)}=\left[p_{i, j}^{(n)}\right]$,

$$
p_{i, j}^{(n)}:=\mathbf{P}\left\{Z_{n}=j \mid Z_{n-1}=i\right\} \geq 0, i, j \in I ; \quad \sum_{j=1}^{m} p_{i, j}^{(n)}=1 .
$$

Let $\left\{X_{n}\right\}_{n \geq 0}$ be a sequence of non-negative valued random variables, in which for each $n \geq 1$, the variables $X_{1}, \cdots, X_{n}$ are $\left\{Z_{0}, \cdots, Z_{n-1}\right\}$-conditionally independent and satisfying

$$
\left.\mathbf{P}\left\{X_{1} \leq x_{1}, \cdots, X_{n} \leq x_{n}\right\} \mid Z_{0}, \cdots Z_{n-1}\right\}=F_{Z_{0}}\left(x_{1}\right) \cdots F_{Z_{n-1}}\left(x_{n}\right),
$$

and $X_{0}=0$. The pair $(Z, X):=\left\{\left(Z_{n}, X_{n}\right)\right\}_{n \geq 0}$ becomes a semi-Markov process on $(\Omega, \mathcal{F}, \mathcal{P})$, since

$$
Q_{i, j}^{(n)}(x):=\mathbf{P}\left\{Z_{n}=j, X_{n} \leq x \mid\left(Z_{k}, X_{k}\right), 0 \leq k \leq n-1\right\}=p_{i, j}^{(n)} F_{i}(x),
$$


is a semi-Markov kernel satisfying

$$
\lim _{x \rightarrow \infty} Q_{i, j}^{(n)}(x)=p_{i, j}^{(n)}, i, j \in I
$$

We remark that in the usual semi-Markov structure, the variable $X_{n}$ may depend not only on $Z_{n-1}$ but also on $Z_{n}$. The following is the direct consequence of Proposition 3.2 in Janssen (2006).

Lemma 2.1. For $i, j \in I$,

$$
\mathbf{P}\left\{X_{n} \leq x \mid Z_{n-1}=i, Z_{n}=j\right\}= \begin{cases}F_{i}(x), & p_{i, j}^{(n)}>0 \\ U(x), & p_{i, j}^{(n)}=0\end{cases}
$$

where $U(x)=0, x<0 ;=1, x \geq 0$.

Now, let us define a sequence $T=\left\{T_{n}\right\}_{n \geq 0}$ of random variables as

$$
T_{0}=0, \quad T_{n}=T_{n-1}+X_{n}, \quad n \geq 1
$$

which is called a renewal point process, and the pair $(Z, T)=\left\{\left(Z_{n}, T_{n}\right)\right\}_{n \geq 0}$ thus defined is called a Markov renewal process. Hereafter, we will abbreviate Markov renewal process to "MRP". It follows from the semi-Markov property that

$$
\begin{aligned}
& \mathbf{P}\left\{Z_{n}=j, T_{n} \leq x \mid\left(Z_{k}, T_{k}\right), k=0, \cdots, n-1\right\} \\
& =\mathbf{P}\left\{Z_{n}=j, X_{n} \leq x-T_{n-1} \mid\left(Z_{k}, T_{k}\right), k=0, \cdots, n-1\right\}=F_{Z_{n-1}}^{(n)}\left(x-T_{n-1}\right)
\end{aligned}
$$

In this case the pair $(\mathbf{p}, Q)$ is also called the MRP kernel, since a MRP will be defined by the pair as well.

\subsection{Counting process}

Let us introduce some counting processes associated with a MRP. One is a counting process which counts the number of visiting to a specific state $i \in I$ up to time $t>0$. Other is a total counting process which counts the number of all renewals up to time $t>0$.

For a Markov renewal process $(Z, T)$ a number of transitions to the state $i \in I$ on $(0, t]$ is given by

$$
N_{i}(t)=\sum_{n=1}^{\infty} \mathbf{1}_{\left\{Z_{n}=i, T_{n} \leq t\right\}}, \quad t>0 .
$$

This counting process $\left\{N_{i}(t), t \geq 0\right\}$ with $N_{i}(0)=0$ will be called an $i$-counting process in this paper. Similarly, the total number of renewals on $(0, t]$ for a renewal process $T$ is expressed by

$$
N(t)=\sum_{n=1}^{\infty} \mathbf{1}_{\left\{T_{n} \leq t\right\}}, \quad t>0 .
$$


The process $\{N(t), t \geq 0\}$ thus defined with $N(0)=0$ will be called a total counting process. It follows from these definitions that

$$
N(t)=\sum_{i=1}^{m} N_{i}(t), \quad t \geq 0 .
$$

\subsection{The Renewal functions}

Generally, an expectation function of a counting process is called as a renewal function in renewal theory. Let us consider the renewal function $H_{i}(t):=\mathbf{E}\left[N_{i}(t)\right]$ of the $i$-counting process. It follows from the expression (9) that

$$
H_{i}(t)=\sum_{n=1}^{\infty} \mathbf{P}\left\{Z_{n}=i, T_{n} \leq t\right\}, \quad t \geq 0 .
$$

By the Markov property of $Z$ and the conditional independence of $X$, we see that

$$
\begin{aligned}
& F_{i}^{n}(t):=\mathbf{P}\left\{Z_{n}=i, T_{n} \leq t\right\} \\
& =\sum_{i_{0}=1}^{m} \sum_{i_{1}=1}^{m} \cdots \sum_{i_{n-1}=1}^{m} \mathbf{P}\left\{Z_{0}=i_{0}, Z_{1}=i_{1}, \cdots, Z_{n}=i, T_{n} \leq t\right\} \\
& =\sum_{i_{0}=1}^{m} \sum_{i_{1}=1}^{m} \cdots \sum_{i_{n-1}=1}^{m} \mathbf{P}\left\{X_{1}+X_{2}+\cdots+X_{n} \leq t \mid Z_{0}=i_{0}, Z_{1}=i_{1}, \cdots, Z_{n}=i\right\} \\
& =\sum_{i_{0}=1}^{m} \sum_{i_{1}=1}^{m} \cdots \sum_{i_{n-1}=1}^{m} p_{i_{0}} p_{i_{0}, i_{1}}^{(1)} \cdots p_{i_{n-1}, i}^{(n)} F_{i_{0}} * F_{i_{1}} * \cdots * F_{i_{n-1}}(t),
\end{aligned}
$$

where the symbole “*” indicates convolution. This gives the following result.

THEOREM 2.2. The i-renewal function is expressed by

$$
H_{i}(t)=\sum_{n=1}^{\infty} F_{i}^{n}(t), \quad t \geq 0,
$$

where $F_{i}^{n}(t)$ is given by (12). Moreover,

$$
H(t)=\sum_{i=1}^{m} H_{i}(t)=\sum_{n=1}^{\infty} \sum_{i=1}^{m} F_{i}^{n}(t), \quad t \geq 0 .
$$

\subsection{Sojourn probability}

Let us consider a sojourn probability which is a probability of a MRP being in a specific state at time $t \geq 0$. Firstly, we consider the following probability,

$$
P_{i}^{n}(t):=\mathbf{P}\left\{Z_{n}=i, T_{n} \leq t<T_{n+1}\right\}
$$


which means a probability of a MRP being in a state $i \in I$ at time $t \geq 0$ after the $n$-th transition. In the same way as (12), we see that

$$
\begin{aligned}
& P_{i}^{n}(t)=\mathbf{P}\left\{Z_{n}=i, T_{n} \leq t\right\}-\mathbf{P}\left\{Z_{n}=i, T_{n+1} \leq t\right\} \\
& =\sum_{i_{0}=1}^{m} \sum_{i_{1}=1}^{m} \cdots \sum_{i_{n-1}=1}^{m} p_{i_{0}} p_{i_{0}, i_{1}}^{(1)} \cdots p_{i_{n-1}, i}^{(n)} F_{i_{0}} * F_{i_{1}} * \cdots * F_{i_{n-1}}(t) \\
& \quad-\sum_{i_{0}=1}^{m} \sum_{i_{1}=1}^{m} \cdots \sum_{i_{n-1}=1}^{m} p_{i_{0}} p_{i_{0}, i_{1}}^{(1)} \cdots p_{i_{n-1}, i}^{(n)} F_{i_{0}} * F_{i_{1}} * \cdots * F_{i_{n-1}} * F_{i}(t),
\end{aligned}
$$

and

$$
P_{i}^{0}(t)=p_{i}\left(1-F_{i}(t)\right)
$$

Thus, we obtain the following result with using Theorem 2.2.

COROLLARY 2.3. A sojourn probability of a MRP being in a state $i \in I$ at time $t \geq 0$ in the $n$-th transition is

$$
P_{i}^{n}(t)=F_{i}^{n}(t)-F_{i}^{n} * F_{i}(t), \quad i \in I,
$$

where $F_{i}^{0}(t)=p_{i}$. Hence, the sojourn probability of a MRP being in a state $i$ at time $t>0$ is

$$
P_{i}(t)=\sum_{n=0}^{\infty} P_{i}^{n}(t), \quad i \in I
$$

Note that

$$
\sum_{i=1}^{m} P_{i}(t)=\sum_{n=0}^{\infty} \mathbf{P}\left\{T_{n} \leq t<T_{n+1}\right\}=1, \quad t \geq 0 .
$$

Remark that the sojourn probabilities show mean transition dynamics of a tire during its lifetime.

\section{Terminating MRP's of finite transition type}

In this section we set up a model for lifetime of a truck's tire by a MRP, which stops at a finite $K \geq 0$ steps. It is a special case of the $D(\alpha, \beta, k)$-terminating MRP, that will be defined in Section 4. A truck tire fitted to some axis of a truck will wear its tread and become bald, and then it will be on the scrap, or it may be retreaded at a factory and will be refitted to some other axis of another truck again. If it is usable other than its tread, then it may be retreaded again and so on. In North America, tires fitted to trucks are usually retreaded once or twice, whereas in Japan only $15 \%$ of truck's tires are retreaded just once. This is the replacement problem which we are going to be in minds. 


\subsection{Lifetime model of a retreadable tire}

In Endow ( 2008) and Endow and Tanimoto (2010), a replacement problem of tires fitted to a motorcar is modeled by a terminal MRP, or a renewal process with termination, because tires fitted to a motorcar are disposed, or on the scrap when they become bald, and new ones are fitted to the motorcar again, and so on. This process continues when the motorcar is disposed itself.

In contrast to the motorcar case a tire fitted to a truck may be retreaded once or twice, so we will set up a lifetime model of a retreadable tire by a MRP which terminates up to a finite fixed number $K$ of transitions, which is one of the $D(\alpha, \beta, k)$ type MRP introduced later. An $i$-counting process and an $i$-renewal function for this terminating MRP, which are the counterparts of (9) and (13), are expressed by

$$
N_{i}(K ; t)=\sum_{n=1}^{K} \mathbf{1}_{\left\{Z_{n}=i, T_{n} \leq t\right\}}, \quad t \geq 0,
$$

and

$$
H_{i}(K ; t)=\sum_{n=1}^{K} F_{i}^{n}(t), \quad i \in I,
$$

respectively. A sojourn probability of the MRP being in a state $i$ at time $t \geq 0$ will be also given by

$$
P_{i}(K ; t)=\sum_{n=0}^{K} P_{i}^{n}(t), \quad i \in I .
$$

\subsection{A lifetime model of truck tires}

Let $I=\{1,2,3,4,5,6,7,8\}$ be a state space, in which the numbers designate as follows;

1 the state of "retread"; R,

2 the state of fitted to "a steering axis"; $\mathrm{S}$,

3 the state of fitted to "a driving axis"; D,

4 the state of fitted to "a trailing axis"; T,

5 the state of fitted to "a steering axis after retreaded"; RS,

6 the state of fitted to "a driving axis after retreaded"; RD,

7 the state of fitted to "a trailing axis after retreaded"; RT,

8 the state of "disposed"; DP. 
Let $\mathbf{p}=\left(0, p_{2}, p_{3}, p_{4}, 0,0,0,0,\right)$ be an initial probability of a Markov process such that $p_{i}>0, i=2,3,4 ; p_{2}+p_{3}+p_{4}=1$. This means a new tire is fitted to one of the axles $\mathrm{S}, \mathrm{D}$, and T. Transition probability matrices $p^{(n)}, 1 \leq n \leq K$ are given by

$$
p^{(n)}=\left[\begin{array}{cccccccc}
0 & 0 & 0 & 0 & p_{1,5} & p_{1,6} & p_{1,7} & p_{1,8} \\
p_{2,1} & 0 & 0 & 0 & 0 & 0 & 0 & p_{2,8} \\
p_{3,1} & 0 & 0 & 0 & 0 & 0 & 0 & p_{3,8} \\
p_{4,1} & 0 & 0 & 0 & 0 & 0 & 0 & p_{4,8} \\
p_{5,1} & 0 & 0 & 0 & 0 & 0 & 0 & p_{5,8} \\
p_{6,1} & 0 & 0 & 0 & 0 & 0 & 0 & p_{6,8} \\
p_{7,1} & 0 & 0 & 0 & 0 & 0 & 0 & p_{7,8} \\
0 & 0 & 0 & 0 & 0 & 0 & 0 & 1
\end{array}\right], \quad 1 \leq n<K,
$$

and

$$
p^{(K)}=\left[\begin{array}{cccccccc}
0 & 0 & 0 & 0 & 0 & 0 & 0 & 1 \\
0 & 0 & 0 & 0 & 0 & 0 & 0 & 1 \\
0 & 0 & 0 & 0 & 0 & 0 & 0 & 1 \\
0 & 0 & 0 & 0 & 0 & 0 & 0 & 1 \\
0 & 0 & 0 & 0 & 0 & 0 & 0 & 1 \\
0 & 0 & 0 & 0 & 0 & 0 & 0 & 1 \\
0 & 0 & 0 & 0 & 0 & 0 & 0 & 1 \\
0 & 0 & 0 & 0 & 0 & 0 & 0 & 1
\end{array}\right]
$$

where

$$
p_{i, j} \geq 0, \quad i, j \in I ; \quad \sum_{j=5}^{8} p_{1, j}=1, p_{i, 1}+p_{i, 8}=1, \quad i=2, \cdots, 7 .
$$

Let $F_{i}(x), i \in I$ be the d.f. of a sojourn time for $i$-state. Putting

$$
Q_{i, j}^{(n)}(t):=p_{i, j}^{(n)} F_{i}(t), \quad i, j \in I, t \geq 0, \quad n=1, \cdots, K,
$$

and

$$
Q^{(n)}(t)=\left[Q_{i, j}^{(n)}(t)\right], \quad t \geq 0, \quad n=1, \cdots, K,
$$

we have a $K$-terminating $\operatorname{MRP}(\mathbf{p}, Q)$, where $Q=\left\{Q^{(n)}\right\}_{1 \leq n \leq K}$. Remark that if we put $P^{(n)}=P^{(K)}, n>K$, then the $K$-terminating MRP thus defined is not substantially different from $\operatorname{MRP}(\mathbf{p}, Q)$, because both of the processes will be stayed at state " 8 " after the $K$-th transition.

\subsubsection{Examples}

EXAmPle 3.1. (Non retread case) Firstly, we consider no retread case, which corresponds to $K=1$. A tire fitted to a motorcar will be disposed when it becomes bald. This can be expressed in terms of a Markov renewal process with one transition. The sojourn probability in the $i$-state at $t \geq 0$ is given by

$$
P_{i}(1 ; t)=P_{i}^{0}(t)= \begin{cases}p_{i}\left(1-F_{i}(t)\right), & i=2,3,4, \\ 0, & i=1,5,6,7, \\ \sum_{i_{0}=2}^{4} p_{i_{0}} F_{i_{0}}(t), & i=8\end{cases}
$$


EXAMPlE 3.2. (Once retreaded case) As stated above in the Japanese truck tires market, $15 \%$ of the truck tires are retreaded up to once. So, three cases are considered; (1) a tire is disposed when it becomes bald, (2) a tire is retreaded when it becomes bald, and it is refitted to some axis and is disposed when it becomes bald again, and (3) a tire is retreaded when it becomes bald, and if it is not passed the quality test it will be disposed. In this case, $K=3$ and the sojourn probability in the $i$-state at $t \geq 0$ is given by

$$
P_{i}(3 ; t)=\sum_{n=0}^{3} P_{i}^{n}(t)= \begin{cases}P_{1}^{1}(t), & i=1 \\ P_{i}^{0}(t), & i=2,3,4 \\ P_{i}^{2}(t), & i=5,6,7 \\ P_{8}^{1}(t)+P_{8}^{2}(t)+P_{8}^{3}(t), & i=8\end{cases}
$$

where

$$
\begin{aligned}
P_{i}^{0}(t)= & p_{i}\left(1-F_{i}(t)\right), \quad i=2,3,4 \\
P_{i}^{1}(t)= & \sum_{i_{0}=2}^{4} p_{i_{0}} P_{i_{0}, i}^{(1)}\left(F_{i_{0}}(t)-F_{i_{0}} * F_{i}(t)\right), \quad i=1,8 \\
P_{i}^{2}(t)= & \sum_{i_{0}=2}^{4} p_{i_{0}} P_{i_{0}, 1}^{(1)} P_{1, i}^{(2)}\left(F_{i_{0}} * F_{1}(t)-F_{i_{0}} * F_{1} * F_{i}(t)\right), \quad i=5,6,7, \\
P_{8}^{2}(t)= & \sum_{i_{0}=2}^{4} p_{i_{0}} P_{i_{0}, 8}^{(1)}\left(F_{i_{0}} * F_{8}(t)-F_{i_{0}} * F_{8} * F_{8}(t)\right) \\
& +\sum_{i_{0}=2}^{4} p_{i_{0}} P_{i_{0}, 1}^{(1)} P_{1,8}^{(2)}\left(F_{i_{0}} * F_{1}(t)-F_{i_{0}} * F_{1} * F_{8}(t)\right), \\
P_{8}^{3}(t)= & \sum_{i_{0}=2}^{4} p_{i_{0}} P_{i_{0}, 8}^{(1)}\left(F_{i_{0}} * F_{8} * F_{8}(t)-F_{i_{0}} * F_{8} * F_{8} * F_{8}(t)\right) \\
& +\sum_{i_{0}=2}^{4} p_{i_{0}} P_{i_{0}, 1}^{(1)} P_{1,8}^{(2)}\left(F_{i_{0}} * F_{1} * F_{8}(t)-F_{i_{0}} * F_{1} * F_{8} * F_{8}(t)\right) \\
& +\sum_{i_{0}=2}^{4} \sum_{i_{2}=5}^{7} p_{i_{0}} P_{i_{0}, 1}^{(1)} P_{1, i_{2}}^{(2)} P_{i_{2}, 8}^{(3)}\left(F_{i_{0}} * F_{1} * F_{i_{2}}(t)-F_{i_{0}} * F_{1} * F_{i_{2}} * F_{8}(t)\right)(34)
\end{aligned}
$$

Let us set up a numerical example of this case as follows. Put $\mathbf{p}=(0,0.2,0.7,0.1,0,0,0,0$,$) ,$ and

$$
p^{(n)}=\left[\begin{array}{cccccccc}
0 & 0 & 0 & 0 & 0.15 & 0.65 & 0.10 & 0.10 \\
0.15 & 0 & 0 & 0 & 0 & 0 & 0 & 0.85 \\
0.15 & 0 & 0 & 0 & 0 & 0 & 0 & 0.85 \\
0.15 & 0 & 0 & 0 & 0 & 0 & 0 & 0.85 \\
0.10 & 0 & 0 & 0 & 0 & 0 & 0 & 0.90 \\
0.10 & 0 & 0 & 0 & 0 & 0 & 0 & 0.90 \\
0.10 & 0 & 0 & 0 & 0 & 0 & 0 & 0.90 \\
0 & 0 & 0 & 0 & 0 & 0 & 0 & 1
\end{array}\right], n=1,2
$$


Terminating Markov renewal processes in view of lifetime model of retreaded tires fitted to trucks 31

and $p^{(3)}$ is equal to the matrix in (25). The sojourn times for $i \in I$ follow the normal distributions with parameters in Table 1. Figure 1 shows the graph of sojourn probabilities of this case.

Table 1: Parameters for $N\left(\mu_{i}, \sigma_{i}^{2}\right)$

\begin{tabular}{ccccccccc}
\hline$i$ & 1 & 2 & 3 & 4 & 5 & 6 & 7 & 8 \\
\hline$\mu_{i}$ & 0.5 & 2.0 & 2.0 & 2.0 & 1.5 & 1.5 & 1.5 & 1000 \\
$\sigma^{2}$ & $0.3^{2}$ & $0.5^{2}$ & $0.5^{2}$ & $0.5^{2}$ & $0.5^{2}$ & $0.5^{2}$ & $0.5^{2}$ & $0.5^{2}$ \\
\hline
\end{tabular}

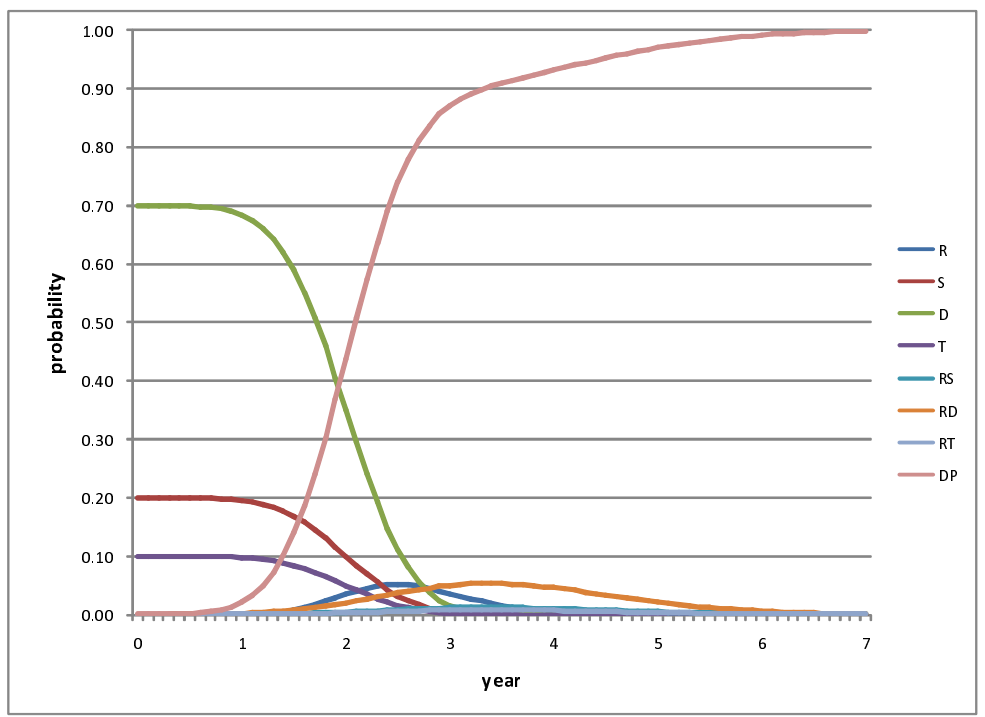

Figure 1: Sojourn probabilities

\section{Terminating MRP's of $D(\alpha, \beta, k)$ type}

In this section, we introduce a termination rule for such a MRP, which will be called $D(\alpha, \beta, k)$ type, and consider its termination time and give simple numerical examples in view of a replacement problem of truck tires. Hereafter, a $\operatorname{MRP}(\mathbf{p}, Q)$ is assumed to be homogeneous, namely, whose semi-Markov kernel does not depend on steps; $Q^{(n)}=Q, n \geq 1$.

\subsection{Definition}

Before introduction of a terminating MRP of $D(\alpha, \beta, k)$ type, some types of termination are reviewed in order. In the following, to avoid cumbersome notations, we do 
not distinguish in notation between the subsets $\alpha, \beta$ and $\gamma$ of the set $I=\{1,2, \ldots, m\}$ and their cardinal numbers, $\alpha, \beta$ and $\gamma$, respectively.

Definition 4.1. (Becker (1982)) The MRP will be of type $A(\alpha)$ if it terminates on first transition to any one of a particular subset of $\alpha$ states.

The MRP will be of type $B\left(\alpha ; k_{1}, k_{2}, \cdots, k_{\alpha}\right)$ if it terminates when the first time transition is made for the $k_{i}$-th consecutive time to the $i$-th state in a subset of $\alpha$ states. Special case of type $B$ termination which will be considered is type $B(\alpha ; k)$ in which $k_{1}=k_{2}=\cdots=k_{\alpha}=k$ and $\alpha<m$.

The MRP will be of type $C\left(\alpha ; k_{1}, k_{2}, \cdots, k_{\alpha}\right)$ if it terminates when the first time transition is made for the $k_{i}$-th times (not necessarily consecutively) to the $i$-th state in a subset of $\alpha$ states. Special case are defined as in type $B$ processes. Thus type $C(\alpha ; k)$ means that $k_{1}=k_{2}=\cdots=k_{\alpha}=k$ and $\alpha<m$.

Definition 4.2. Let $I=\{1,2, \ldots, m\}$ be the state space, and the subsets $\alpha, \beta, \gamma$ constitute a partition of $I$, such that $\gamma=\{1,2, \cdots, \gamma\}, \alpha=\{\gamma+1, \gamma+2, \cdots, \gamma+\alpha\}$ and $\beta=\{\gamma+\alpha+1, \gamma+\alpha+2, \cdots, m\}$. The MRP will be of type $D(\alpha, \beta, \gamma, k),(k=2,3, \cdots)$, if it terminates when the first time transition is made for the $k$-th times to the state of $\gamma$, which starts from a state in $\alpha$ firstly and translate to a state in $\gamma$, then translate a state in $\beta$, and translate to a state in $\gamma$ again, for example

$$
Z_{0}=\alpha_{0} \longrightarrow \gamma_{1} \longrightarrow \beta_{1} \longrightarrow \gamma_{2} \longrightarrow \beta_{2} \longrightarrow \cdots \longrightarrow \beta_{k-1} \longrightarrow \gamma_{k}=Z_{2 k-1}
$$

in which, $\alpha_{0} \in \alpha, \gamma_{1}, \gamma_{2}, \cdots, \gamma_{k} \in \gamma$, and $\beta_{1}, \beta_{2}, \cdots, \beta_{k-1} \in \beta$.

In the Definition 4.2 , if the subset $\gamma$ only has one state, that is $\gamma=\{1\}$, then this type termination will denoted by $D(\alpha, \beta, k)$ for short. Notice that the terminating MRP of type $D(\alpha, \beta, \gamma, k)$ will stop just after the $2 k-1$ transitions if the stopping rule is satisfied, otherwise it will not stop forever.

Whatever the form of termination, two random variables associated with termination, will be important, i.e., (a) the variable $N_{\tau}=k$, where $k$ is the number of transitions to termination, and (b) the variable $\tau=X_{1}+X_{2}+\cdots+X_{k}$ which is the actual time to termination. Let the Laplace-Stieltjes Transform (L-S.T.) of a semi-Markov kernel $Q=Q(x)=\left[Q_{i j}(x)\right]_{m \times m}$ be denoted by $q(s)=\left[q_{i j}(s)\right]_{m \times m}$, where

$$
q_{i j}(s)=\int_{0}^{\infty} \mathrm{e}^{-s x} \mathrm{~d} Q_{i j}(x), \quad s \geq 0 .
$$

We also denote the corresponding transition matrix by $p_{0}=\left[p_{i j}\right]_{m \times m}$. Then, we see that

$$
I-q(s)=I-p_{0}+s p_{1}-\frac{s^{2}}{2 !} p_{2}+\frac{s^{3}}{3 !} p_{3}-\cdots, \quad s \geq 0
$$

in which

$$
p_{k}=\int_{0}^{\infty} x^{k} \mathrm{~d} Q(x), \quad k=0,1,2, \cdots
$$

assuming all moments exist. 
Terminating Markov renewal processes in view of lifetime model of retreaded tires fitted to trucks 33

REMARK. It follows from (4) and (37) that

$$
p_{k}=\left[p_{i, j} \int_{0}^{\infty} x^{k} \mathrm{e}^{-s x} \mathrm{~d} F_{i}(x)\right]_{m \times m}, \quad k=0,1,2, \cdots,
$$

and

$$
\begin{aligned}
& p_{0}=\left[p_{i, j}\right]_{m \times m}, \\
& p_{1}=\left[p_{i, j} u_{i}\right]_{m \times m}, \\
& p_{2}=\left[p_{i, j}\left(u_{i}^{2}+\sigma_{i}^{2}\right)\right]_{m \times m} . \\
& p_{3}=\left[p_{i, j}\left(u_{i}^{3}+3 \sigma_{i}^{2} u_{i}+\nu_{i}\right)\right]_{m \times m},
\end{aligned}
$$

where $u_{i}, \sigma_{i}^{2}$ and $\nu_{i}$ are the mean, variance and the third central moment (all assumed to be finite) for the d.f. $F_{i}$, respectively.

The $m \times 1$ column vector of zero elements except for one in the $i$-th position will be represented by $a_{i}$. Also, $b_{\alpha}=\sum_{i=1}^{\alpha} a_{i}$.

Some cases of type $A(\alpha), B(\alpha ; k)$, and $C(\alpha ; k)$ MRPs were discussed by Becker (1982). The following discussion is mainly of type $D(\alpha, \beta, k)$ termination.

\subsection{Termination time of type $D(\alpha, \beta, k)$}

Of interest in these MRPs are the time $\tau$ until termination and the number of transition $N_{\tau}$ until termination. For type $D(\alpha, \beta, k)$ termination occurs at the $N_{\tau}=$ $2 k-1(k=2,3, \cdots)$. In this subsection, we try to obtain the distribution and moments of $\tau$.

TheOREM 4.3. For the type $D(\alpha, \beta, k)$, the mean time to termination, conditional on $Z_{0}=i$, is

$$
\begin{array}{r}
u_{\tau \mid i}=a_{i}^{\prime}\left[\sum_{l=0}^{k-2}\left(\widetilde{1}_{1_{0}} p_{0}\right)^{k-l-2}\left(\widetilde{1}_{1}{ }_{1 \alpha} p_{0}+\widetilde{{ }_{1} p_{0 \alpha}} p_{1}\right)\left(\widetilde{1}_{p_{0 \alpha}} p_{0}\right){ }_{1}^{l} \widetilde{p}_{0}+\left(\widetilde{1}_{p_{0 \alpha}} p_{0}\right)^{k-1} \widetilde{ }_{1} \bar{p}_{0}\right] a_{1}, \\
k=2,3, \cdots,
\end{array}
$$

and the variance of the time to termination, conditional on $Z_{0}=i$, is

$$
\begin{aligned}
& \sigma_{\tau \mid i}^{2}
\end{aligned}
$$

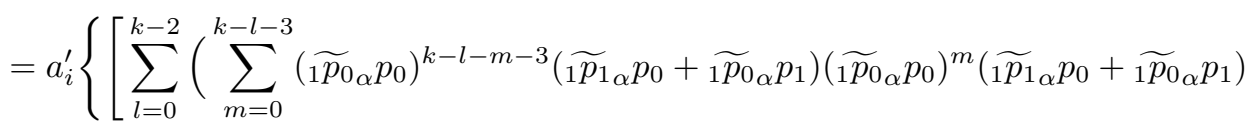

$$
\begin{aligned}
& \times\left(\widetilde{ }_{1 p_{0}} p_{0}\right)^{l}+\left(\widetilde{ }_{1 p_{0}} p_{0}\right)^{k-l-2}\left(\widetilde{ }_{1} \widetilde{p}_{\alpha} p_{0}+2 \widetilde{{ }_{1} p_{1}} p_{1}+\widetilde{1}_{p_{0}} p_{2}\right)\left(\widetilde{ }_{1} \widetilde{p}_{0} p_{0}\right)^{l} \\
& +\sum_{m=0}^{l-1}\left(\widetilde{1}_{p_{0}} p_{0}\right)^{k-l-2}\left(\widetilde{ }_{1} p_{1} p_{0}+\widetilde{ }_{1} \widetilde{p}_{\alpha} p_{1}\right)\left(\widetilde{1}_{p_{0}} p_{0}\right)^{l-m-1}\left(\widetilde{1}_{p_{1}} p_{0}+\widetilde{ }_{1}{p_{0}}_{\alpha} p_{1}\right) \\
& \left.\left.\times\left(\widetilde{ }_{1} p_{0} p_{0}\right)^{m}\right)\right] \widetilde{{ }_{1} p_{0}}+2 \sum_{l=0}^{k-2}\left(\widetilde{1}_{1} p_{0} p_{0}\right)^{k-l-2}\left(\widetilde{ }_{1} \widetilde{p}_{1_{\alpha}} p_{0}+\widetilde{ }_{1 p_{0}} p_{1}\right)\left(\widetilde{1}_{1} p_{0} p_{0}\right)^{l} \widetilde{1}_{1} \\
& \left.+\left(\widetilde{ }_{1 p_{0 \alpha}} p_{0}\right)^{k-1} \underset{{ }_{1} \widetilde{p}_{2}}{ }\right\} a_{1}-u_{\tau \mid i}^{2}, \quad k=3,4, \cdots,
\end{aligned}
$$




$$
\begin{aligned}
& \sigma_{\tau \mid i}^{2}=a_{i}^{\prime}\left[\widetilde{1}_{p_{2}} p_{01} \widetilde{p_{0}}+\widetilde{1}_{{ }_{0}}{ }_{0} p_{21} \widetilde{p}_{0}+{ }_{1{ }_{0}}{ }_{0 \alpha} p_{01} \widetilde{p_{2}}\right. \\
& \left.+2\left(\widetilde{1}_{1} p_{\alpha} p_{11} \widetilde{p}_{0}+\widetilde{ }_{1 p_{1}} p_{01} \widetilde{p}_{0}+{ }_{{ }_{1} p_{0}} p_{11} \widetilde{p}_{0}\right)\right] a_{1}-u_{\tau \mid i}^{2}, \quad k=2,
\end{aligned}
$$

where ${ }_{\alpha} p_{e}$ is the matrix $p_{e}$ with the first $\alpha+1$ columns replaced by zeros, and $\widetilde{{ }_{1} p_{e}}$ is the matrix $p_{e}$ with the last $m-1$ columns replaced by zeros.

Proof: By considering the various paths to termination, the joint distribution function of $\left(\tau, N_{\tau}\right)$, conditional on $Z_{0}=i$, is given by

$$
\begin{aligned}
& L_{i}(u, k ; \alpha):=\mathbf{P}\left\{\tau \leq u, N_{\tau}=2 k-1 \mid Z_{0}=i\right\} \\
& \quad=\sum_{j_{1}=\alpha+2}^{m} \cdots \sum_{j_{k-1}=\alpha+2}^{m} Q_{i 1} * Q_{1 j_{1}} * Q_{j_{1} 1} * Q_{1 j_{2}} * Q_{j_{2} 1} * \cdots * Q_{1 j_{k-1}} * Q_{j_{k-1} 1}(u),
\end{aligned}
$$

where $i=2,3, \cdots, \alpha+1$. The L-S.T. of this function, with respect to $u$, is also given by,

$$
l_{i}(s, k ; \alpha)=\sum_{j_{1}=\alpha+2}^{m} \cdots \sum_{j_{k-1}=\alpha+2}^{m} q_{i 1} q_{1 j_{1}} q_{j_{1} 1} q_{1 j_{2}} q_{j_{2} 1} \cdots q_{1 j_{k-1}} q_{j_{k-1} 1}(s),
$$

where $q_{\alpha}$ is the matrix $q$ with the first $\alpha+1$ columns replaced by zeros, and $\widetilde{q_{1}}$ is the matrix $q$ with the last $m-1$ columns replaced by zeros. By the multiplication of matrices, in matrix notation, we can rewrite (43) as

$$
\begin{aligned}
l_{i}(s, k ; \alpha) & =a_{i}^{\prime}\left[\widetilde{q_{1}} q_{\alpha} \widetilde{q_{1}} q_{\alpha} \cdots \widetilde{q_{1}} q_{\alpha} \widetilde{q_{1}}\right] a_{1} \\
& =a_{i}^{\prime}\left[\left(\widetilde{q_{1}} q_{\alpha}\right)\left(\widetilde{q_{1}} q_{\alpha}\right) \cdots\left(\widetilde{q_{1}} q_{\alpha}\right) \widetilde{q_{1}}\right] a_{1} \\
& =a_{i}^{\prime}\left[\left(\widetilde{q_{1}} q_{\alpha}\right)^{k-1} \widetilde{q_{1}}\right] a_{1},
\end{aligned}
$$

which means the L-S.T. of the d.f. of time to termination $\tau$, conditional on $Z_{0}=i$.

Let $\widetilde{q_{1}}:=f(s), q_{\alpha}:=g(s)$ and $F_{1}(s):=(f(s) g(s))^{k-1}$, then

$$
\begin{aligned}
F_{1}^{\prime}(s)= & \sum_{l=0}^{k-2}(f(s) g(s))^{k-l-2}(f(s) g(s))^{\prime}(f(s) g(s))^{l} \\
F_{1}^{\prime \prime}(s)= & \sum_{l=0}^{k-2}\left(\sum_{m=0}^{k-l-3}(f g)^{k-l-m-3}(f g)^{\prime}(f g)^{m}(f g)^{\prime}(f g)^{l}+(f g)^{k-l-2}(f g)^{\prime \prime}(f g)^{l}\right. \\
& \left.\quad+\sum_{m=0}^{l-1}(f g)^{k-l-2}(f g)^{\prime}(f g)^{l-m-1}(f g)^{\prime}(f g)^{m}\right) .
\end{aligned}
$$

Let $F(s):=\left(\widetilde{q_{1}} q_{\alpha}\right)^{k-1} \widetilde{q_{1}}=(f(s) g(s))^{k-1} f(s)=F_{1}(s) f(s)$, then

$$
\begin{gathered}
F^{\prime}(0)=F_{1}^{\prime}(0) f(0)+F_{1}(0) f^{\prime}(0), \\
F^{\prime \prime}(0)=F_{1}^{\prime \prime}(0) f(0)+2 F_{1}^{\prime}(0) f^{\prime}(0)+F_{1}(0) f^{\prime \prime}(0),
\end{gathered}
$$


Terminating Markov renewal processes in view of lifetime model of retreaded tires fitted to trucks 35

and

$$
\begin{array}{lll}
f(0)=\widetilde{{ }_{1} p_{0}}, & f^{\prime}(0)=\widetilde{{ }_{1} p_{1}}, & f^{\prime \prime}(0)=\widetilde{{ }_{1} p_{2}}, \\
g(0)={ }_{\alpha} p_{0}, & g^{\prime}(0)={ }_{\alpha} p_{1}, & g^{\prime \prime}(0)={ }_{\alpha} p_{2} .
\end{array}
$$

It follows that the mean time to termination, conditional on $Z_{0}=i$, is

$$
\begin{aligned}
& u_{\tau \mid i}=\left.\frac{\mathrm{d}}{\mathrm{d} s} l_{i}(s, k ; \alpha)\right|_{s=0}=a_{i}^{\prime}\left(\left.\frac{\mathrm{d}}{\mathrm{d} s} F(s)\right|_{s=0}\right) a_{1}=a_{i}^{\prime} F^{\prime}(0) a_{1} \\
& =a_{i}^{\prime}\left[\sum_{l=0}^{k-2}(f g)^{k-l-2}\left(f^{\prime} g+f g^{\prime}\right)(f g)^{l} f+(f g)^{k-1} f\right] a_{1} \text {, }
\end{aligned}
$$

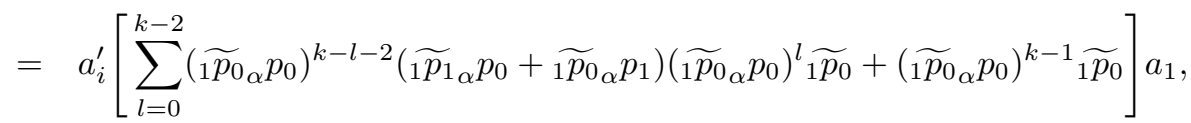

$$
\begin{aligned}
& k=2,3, \cdots,
\end{aligned}
$$

and

$$
\begin{aligned}
& \sigma_{\tau \mid i}^{2}=\left.\frac{\mathrm{d}^{2}}{\mathrm{~d} s^{2}} l_{i}(s, k ; \alpha)\right|_{s=0}-u_{\tau \mid i}^{2}=a_{i}^{\prime} F^{\prime \prime}(0) a_{1}-u_{\tau \mid i}^{2} \\
& =a_{i}^{\prime}\left\{\left[\sum _ { l = 0 } ^ { k - 2 } \left(\sum_{m=0}^{k-l-3}(f g)^{k-l-m-3}\left(f^{\prime} g+f g^{\prime}\right)(f g)^{m}\left(f^{\prime} g+f g^{\prime}\right)(f g)^{l}+(f g)^{k-l-2}\right.\right.\right. \\
& \times\left(f^{\prime \prime} g+2 f^{\prime} g^{\prime}+f g^{\prime \prime}\right)(f g)^{l}+\sum_{m=0}^{l-1}(f g)^{k-l-2}\left(f^{\prime} g+f g^{\prime}\right)(f g)^{l-m-1}\left(f^{\prime} g+f g^{\prime}\right) \\
& \left.\left.\left.\times(f g)^{m}\right)\right] f+2 \sum_{l=0}^{k-2}(f g)^{k-l-2}\left(f^{\prime} g+f g^{\prime}\right)(f g)^{l} f^{\prime}+(f g)^{k-1} f^{\prime \prime}\right\} a_{1}-u_{\tau \mid i}^{2} .
\end{aligned}
$$

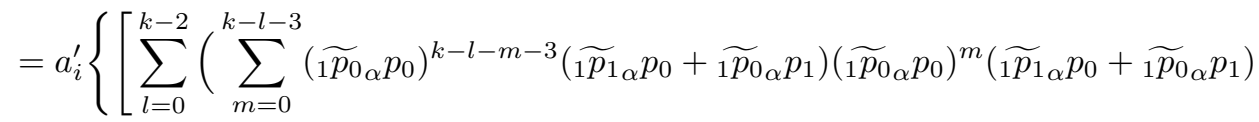

$$
\begin{aligned}
& \times\left(\widetilde{ }_{1} p_{0} p_{0}\right)^{l}+\left(\widetilde{ }_{1} p_{0} p_{0}\right)^{k-l-2}\left(\widetilde{1}_{p_{2}} p_{0}+2 \widetilde{1}_{1} \widetilde{p}_{1} p_{1}+{ }_{1} \widetilde{p}_{0 \alpha} p_{2}\right)\left(\widetilde{1}_{p_{0}} p_{0}\right)^{l}
\end{aligned}
$$

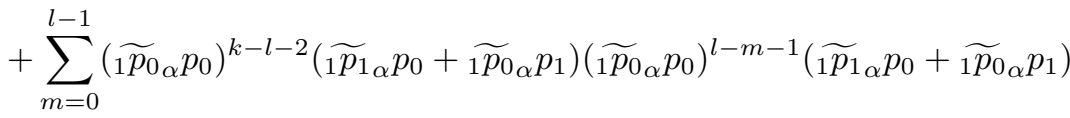

$$
\begin{aligned}
& \left.\left.\times\left(\widetilde{ }_{1} p_{0} p_{0}\right)^{m}\right)\right] \widetilde{p_{0}}+2 \sum_{l=0}^{k-2}\left(\widetilde{1}_{p_{0}} p_{0}\right)^{k-l-2}\left(\widetilde{ }_{1} p_{1} p_{0}+{ }_{1} p_{0}{ }_{\alpha} p_{1}\right)\left({ }_{1} p_{0}{ }_{\alpha} p_{0}\right) l{ }_{1} \widetilde{p}_{1} \\
& \left.+\left(\widetilde{ }_{1 p_{0}} p_{0}\right)^{k-1} \underset{{ }_{1} \widetilde{p}_{2}}{\widetilde{1}}\right\} a_{1}-u_{\tau \mid i}^{2} . \quad k=3,4, \cdots,
\end{aligned}
$$

for $k=2$,

$$
\begin{aligned}
\sigma_{\tau \mid i}^{2}= & a_{i}^{\prime}\left[\widetilde{{ }_{1} p_{2}} p_{01} \widetilde{p_{0}}+\widetilde{{ }_{1} p_{0 \alpha}} p_{21} \widetilde{p_{0}}+\widetilde{{ }_{1} p_{0 \alpha}} p_{01} \widetilde{p_{2}}\right. \\
& \left.+2\left(\widetilde{ }_{1 p_{1}} p_{11} \widetilde{p_{0}}+\widetilde{{ }_{1} p_{1 \alpha}} p_{01} \widetilde{p_{0}}+\widetilde{{ }_{1} p_{0 \alpha}} p_{11} \widetilde{p_{0}}\right)\right] a_{1}-u_{\tau \mid i}^{2},
\end{aligned}
$$

which completes the proof of Theorem 4.3. 
Remark. From Definition 4.1 and Definition 4.2, the type $D(\alpha, \beta, k)$ can be look as a special kind of type $C(\alpha ; k)$. The various kind of type $C$ terminating processes lead to cumbersome expressions for the joint distribution of $\left(\tau, N_{\tau}\right)$. These do not facilitate calculation of the moments, so Becker (1982) just discussed about the case of type $C(1,2)$, only. But for the type $D(\alpha, \beta, k)$, Theorem 4.3 gives the moments for every $k$, explicitly.

To illustrate one of the result of above Theorem 4.3 for the type of $D(\alpha, \beta, k)$, we give an example bellow.

Example 4.4. Consider the simple model of $D(\alpha, \beta, 2)$ with $I=\{1,2, \ldots, 5\}$ states. Let $\alpha=\{2,3\}, \beta=\{4,5\}$, and $Z_{0}=2$. By definitions (39)-(42) we see that

$$
\begin{aligned}
& p_{0}=\left[\begin{array}{ccccc}
0 & 0 & 0 & a & b \\
1 & 0 & 0 & 0 & 0 \\
1 & 0 & 0 & 0 & 0 \\
1 & 0 & 0 & 0 & 0 \\
1 & 0 & 0 & 0 & 0
\end{array}\right], \quad p_{1}=\left[\begin{array}{ccccc}
0 & 0 & 0 & a \cdot u_{1} & b \cdot u_{1} \\
u_{2} & 0 & 0 & 0 & 0 \\
u_{3} & 0 & 0 & 0 & 0 \\
u_{4} & 0 & 0 & 0 & 0 \\
u_{5} & 0 & 0 & 0 & 0
\end{array}\right] \\
& p_{2}=\left[\begin{array}{ccccc}
0 & 0 & 0 & a\left(u_{1}^{2}+\sigma_{1}^{2}\right) & b\left(u_{1}^{2}+\sigma_{1}^{2}\right) \\
u_{2}^{2}+\sigma_{2}^{2} & 0 & 0 & 0 & 0 \\
u_{3}^{2}+\sigma_{3}^{2} & 0 & 0 & 0 & 0 \\
u_{4}^{2}+\sigma_{4}^{2} & 0 & 0 & 0 & 0 \\
u_{5}^{2}+\sigma_{5}^{2} & 0 & 0 & 0 & 0
\end{array}\right] \\
& { }_{2} p_{0}=\left[\begin{array}{ccccc}
0 & 0 & 0 & a & b \\
0 & 0 & 0 & 0 & 0 \\
0 & 0 & 0 & 0 & 0 \\
0 & 0 & 0 & 0 & 0 \\
0 & 0 & 0 & 0 & 0
\end{array}\right], \quad{ }_{2} p_{1}=\left[\begin{array}{ccccc}
0 & 0 & 0 & a \cdot u_{1} & b \cdot u_{1} \\
0 & 0 & 0 & 0 & 0 \\
0 & 0 & 0 & 0 & 0 \\
0 & 0 & 0 & 0 & 0 \\
0 & 0 & 0 & 0 & 0
\end{array}\right] \text {, } \\
& { }_{1} \widetilde{p}_{0}=\left[\begin{array}{ccccc}
0 & 0 & 0 & 0 & 0 \\
1 & 0 & 0 & 0 & 0 \\
1 & 0 & 0 & 0 & 0 \\
1 & 0 & 0 & 0 & 0 \\
1 & 0 & 0 & 0 & 0
\end{array}\right], \quad \widetilde{{ }_{1}}{ }_{1}=\left[\begin{array}{ccccc}
0 & 0 & 0 & 0 & 0 \\
u_{2} & 0 & 0 & 0 & 0 \\
u_{3} & 0 & 0 & 0 & 0 \\
u_{4} & 0 & 0 & 0 & 0 \\
u_{5} & 0 & 0 & 0 & 0
\end{array}\right] \\
& { }_{2} p_{2}=\left[\begin{array}{ccccc}
0 & 0 & 0 & a\left(u_{1}^{2}+\sigma_{1}^{2}\right) & b\left(u_{1}^{2}+\sigma_{1}^{2}\right) \\
0 & 0 & 0 & 0 & 0 \\
0 & 0 & 0 & 0 & 0 \\
0 & 0 & 0 & 0 & 0 \\
0 & 0 & 0 & 0 & 0
\end{array}\right] \text {, }
\end{aligned}
$$

and

$$
\widetilde{1 p_{2}}=\left[\begin{array}{ccccc}
0 & 0 & 0 & 0 & 0 \\
u_{2}^{2}+\sigma_{2}^{2} & 0 & 0 & 0 & 0 \\
u_{3}^{2}+\sigma_{3}^{2} & 0 & 0 & 0 & 0 \\
u_{4}^{2}+\sigma_{4}^{2} & 0 & 0 & 0 & 0 \\
u_{5}^{2}+\sigma_{5}^{2} & 0 & 0 & 0 & 0
\end{array}\right]
$$


From (49), (52)-(57), the mean time to termination is

$$
\begin{aligned}
u_{\tau \mid 2} & =a_{2}^{\prime}\left[\widetilde{1}_{1} \cdot_{2} p_{0} \cdot \widetilde{p_{0}}+\widetilde{ }_{1} p_{0} \cdot 2 p_{1} \cdot \widetilde{p_{0}}+\widetilde{1}_{1} \widetilde{p}_{0} \cdot 2 p_{0} \cdot \widetilde{1}_{1}\right] a_{1} \\
& =a\left(u_{2}+u_{1}+u_{4}\right)+b\left(u_{2}+u_{1}+u_{5}\right) \\
& =u_{2}+a\left(u_{1}+u_{4}\right)+b\left(u_{1}+u_{5}\right),
\end{aligned}
$$

and the variance in the time to termination is

$$
\begin{aligned}
& \sigma_{\tau \mid 2}^{2}=a_{2}^{\prime}\left[\widetilde{1}_{p_{2}} \cdot{ }_{2} p_{0} \cdot \widetilde{1}_{p_{0}}+2 \widetilde{p_{1}} \cdot{ }_{2} p_{1} \cdot \widetilde{1}_{1}+\widetilde{p}_{1} \widetilde{p}_{1} \cdot{ }_{2} p_{0} \cdot \widetilde{1}_{1}\right. \\
& \left.+{ }_{1} p_{0} \cdot 2 p_{2} \cdot{ }_{1} p_{0}+2 \widetilde{p}_{1} \cdot p_{2} p_{1} \cdot \widetilde{{ }_{1} p_{1}}+{ }_{1} p_{0} \cdot 2 p_{0} \cdot \widetilde{p_{2}}\right] a_{1}-u_{\tau \mid 2}^{2} \\
& =u_{2}^{2}+\sigma_{2}^{2}+a\left(u_{1}^{2}+\sigma_{1}^{2}\right)+b\left(u_{1}^{2}+\sigma_{1}^{2}\right)+2 u_{2}\left[a\left(u_{1}+u_{4}\right)+b\left(u_{1}+u_{5}\right)\right] \\
& +2\left(a u_{1} u_{4}+b u_{1} u_{5}\right)-u_{\tau \mid 2}^{2} \\
& =\sigma_{2}^{2}+a\left(\sigma_{1}^{2}+\sigma_{4}^{2}\right)+b\left(\sigma_{1}^{2}+\sigma_{5}^{2}\right)+a\left(u_{1}^{2}+u_{4}^{2}\right)+b\left(u_{1}^{2}+u_{5}^{2}\right) \\
& +2\left(a u_{1} u_{4}+b u_{1} u_{5}\right)-\left[a\left(u_{1}+u_{4}\right)+b\left(u_{1}+u_{5}\right)\right]^{2} \text {. }
\end{aligned}
$$

\subsection{An application for the replacement of truck tires and numerical exam- ples}

In this subsection we model a lifetime of a truck's tire again by terminating MRP of $D(\alpha, \beta, k)$ type, which stops at a finite $k$ steps. We assumed that all tires fitted to trucks are retreaded once or twice, and we try to get the mean and variance of lifetime $\tau$ of truck tires. Let $I=\{1,2,3,4,5,6,7\}$ be a state space, in which the numbers designate the same states as in Section 3.2. without 8 , respectively. Let $\mathbf{p}=\left(0, p_{2}, p_{3}, p_{4}, 0,0,0,\right)$ be the initial probability of a Markov process with $p_{i}>0, i=2,3,4 ; p_{2}+p_{3}+p_{4}=1$. This means a new tire is fitted to one of the axes $\mathrm{S}, \mathrm{D}$, and T. A transition probability matrices $p_{0}$ are given by

$$
p_{0}=\left[\begin{array}{ccccccc}
0 & 0 & 0 & 0 & p_{1,5} & p_{1,6} & p_{1,7} \\
1 & 0 & 0 & 0 & 0 & 0 & 0 \\
1 & 0 & 0 & 0 & 0 & 0 & 0 \\
1 & 0 & 0 & 0 & 0 & 0 & 0 \\
1 & 0 & 0 & 0 & 0 & 0 & 0 \\
1 & 0 & 0 & 0 & 0 & 0 & 0 \\
1 & 0 & 0 & 0 & 0 & 0 & 0
\end{array}\right]
$$

where

$$
p_{i, j} \geq 0, \quad i, j \in I ; \quad \sum_{j=5}^{7} p_{1, j}=1 .
$$

Let $F_{i}(x), i \in I$ be the d.f.s corresponding to the $i$-state. Putting

$$
Q_{i, j}(x):=p_{i, j} F_{i}(x), \quad t \geq 0, i, j \in I
$$

we have a Markov renewal process $(\mathbf{p}, Q)$, where $Q:=\left[Q_{i, j}\right]$. 
Table 2: Parameters for $N\left(\mu_{i}, \sigma_{i}^{2}\right)$

\begin{tabular}{cccccccc}
\hline$i$ & 1 & 2 & 3 & 4 & 5 & 6 & 7 \\
\hline$\mu_{i}$ & 0.5 & 2 & 2.5 & 3 & 1.5 & 1.6 & 1.7 \\
$\sigma_{i}^{2}$ & $0.3^{2}$ & $0.5^{2}$ & $0.6^{2}$ & $0.7^{2}$ & $0.35^{2}$ & $0.4^{2}$ & $0.45^{2}$ \\
\hline
\end{tabular}

Example 4.5. (Numerical examples) Let the subsets be $\alpha=\{2,3,4\}, \beta=\{5,6,7\}$ and the transition probabilities $p_{1,5}=0.15, \quad p_{1,6}=0.65$, and $p_{1,7}=0.2, p_{i j}=$ 0 (otherwise). Assume that the sojourn time obeys normal distribution $N\left(\mu_{i}, \sigma_{i}^{2}\right), i=$ $1, \cdots, 7$ with parameters as in Table 2 . Firstly, we consider once retread case $D(\alpha, \beta, 2)$ with $k=2$ in Theorem 4.3. In Japanese market tires of trucks are retreaded up to once as stated above. The means and variances of lifetime $\tau$ of truck tires obtained by Theorem 4.3 and the numerical results are shown for each starting axis or state in Table 3. The means and the standard deviations are similar to the empirical data that are accepted to some demand prediction experts of truck tires in Bridgestone Company.

Table 3: The mean and variance of lifetime $\tau$ of once retread case

\begin{tabular}{cccc}
\hline $\mathrm{i}$ (state) & 2 (steering axis) & 3 (driving axis) & 4 (trailing axis) \\
\hline$u_{\tau \mid i}$ & 4.11 & 4.61 & 5.11 \\
$\sigma_{\tau \mid i}^{2}$ & $0.71^{2}$ & $0.79^{2}$ & $0.86^{2}$ \\
\hline
\end{tabular}

Similarly, we consider twice retread case $D(\alpha, \beta, 3)$. Tires of trucks are retreaded up to twice or sometimes thrice in the north America. The means and variances of lifetime $\tau$ of truck tires obtained by Theorem 4.3 are shown for each starting axis in Table 4.

Table 4: The mean and variance of lifetime $\tau$ twice retreaded case

\begin{tabular}{cccc}
\hline $\mathrm{i}$ (state) & 2 (steering axis) & 3 (driving axis) & 4 (trailing axis) \\
\hline$u_{\tau \mid i}$ & 6.21 & 6.71 & 7.21 \\
$\sigma_{\tau \mid i}^{2}$ & $0.87^{2}$ & $0.93^{2}$ & $1.00^{2}$ \\
\hline
\end{tabular}

\section{Concluding remarks}

In contrast to the motorcar case a tire fitted to a truck may be retreaded a few times, so we set up a lifetime model of a retreadable tire by a MRP which terminates up to the $K$-th transition. We illustrate the dynamical sojourn probability of this MRP for each Markov state shown in Figure 1. With using these probabilities we will be able to 
set up a couple of prediction models for yearly demand of new truck tires, and retread tires as well.

We also introduce the $D(\alpha, \beta, k)$-terminating Markov renewal process, that includes the $K$-th terminating MRP as a special case. We study fundamental properties of the process such as the time $\tau$ until termination, the distribution and the moments of $\tau$. Theorem 4.3 for the type $D(\alpha, \beta, k)$ gives the mean $u_{\tau \mid i}$ and the variance $\sigma_{\tau \mid i}^{2}$ for any $k \geq 2$, explicitly. The MRP of type $D(\alpha, \beta, k)$ is a generalization of the type $C(\alpha ; k)$, and Becker (1982) discussed about the type $C(1,2)$ only.

We also give some numerical examples in order to check applicability to a lifetime model of retreadable tires fitted to trucks. Among others, Example 3.2 and Example 4.5 give us satisfactory results received recognition of some experts and specialists in Bridgestone Corporation, which is a major tire maker in Japan and in the world.

At the end we would like to add some comments on parameter identification of the MRP modeling a lifetime of truck tires in the markets. For a $\operatorname{MRP}(\mathbf{p}, Q)$, it suffices to set an initial vector $\mathbf{p}$, a transition matrix $P$ of the corresponding embedded $\mathrm{MC}$, and sojourn time distribution functions $F$ 's. In Japanese market, the initial probabilities can be calculated by the yearly data of trucks registered in Ministry of Land, Infrastructure, Transport and Tourism, from which the numbers of tires fitted to each axes, i.e., steering axes, driving axes, and trailing axes, can be obtain, respectively. The transition probabilities and the sojourn time distribution functions are estimated statistically by sampling data conducting a follow-up for transitions of each tire until discarded. However, to collect such data it will take several years on account of Figure 1, so we might as well go some other way at the moment.

\section{Acknowledgement}

The authors would like to thank the anonymous reviewers for their constructive comments improving the paper.

\section{References}

Becker, M. and Kshirsagar, A. H. (1982). Terminating Markov renewal processes. Communications in Statistics-Theory and Methods 11(3): 325-342.

Endow, Y. ( 2008). Renewal systems with termination: An application to demand prediction of replaced tires in the Japanese motorcar market. In Cybernetics and Systems 2008. Proceedings of the Nineteenth European Meeting on Cybernetics and Systems Research, Vienna,Austria. 1: 35-40.

Endow, Y. and Tanimoto, S. ( 2010). A demand prediction model for replacement parts using a markov renewal process with termination. Cybernetics and Systems: An International Journal 41: 287-306.

Janssen, J. and Manca, R. (2006). Applied semi-markov processes Springer. 